
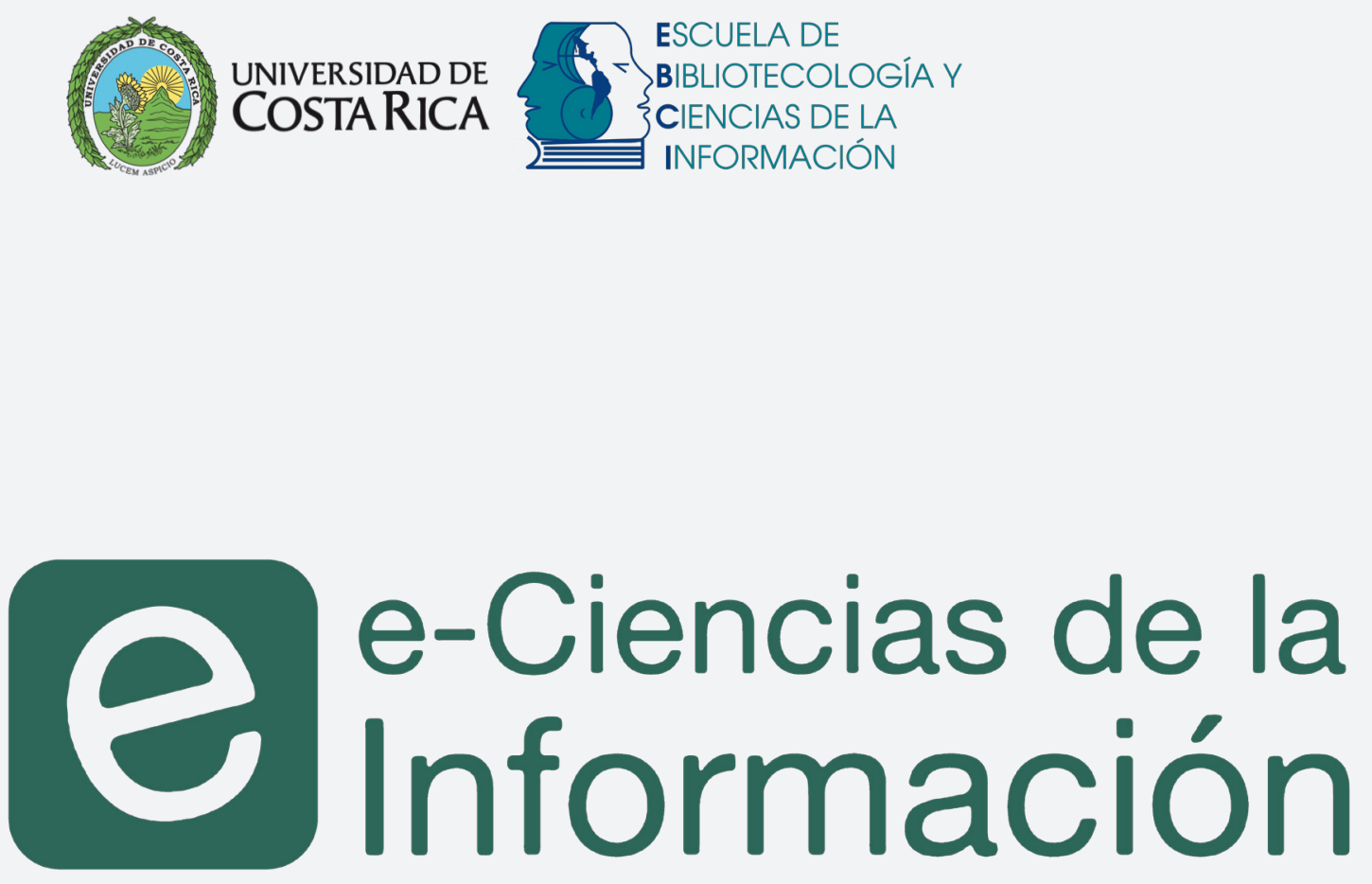

\title{
Los juegos cooperativos y el acceso a la información desde la extensión universitaria Loirette Calvo Sánchez
}

DOI: $\underline{\text { https://doi.org/10.15517/eci.v8i1.30562 }}$

e-Ciencias de la Información, volumen 8, número 1, Ene-Jun 2017

ISSN: $1649-4142$

\section{다(1)(5)}




\section{Los juegos cooperativos y el acceso a la información desde la extensión universitaria}

\section{Cooperative Games and Access to Information from the University Extension}

Loirette Calvo Sánchez'

\section{RESUMEN}

Desde el proyecto de extensión universitaria "Conocimiento, Paz y Gestión Social" durante el mes de noviembre del 2016 se desarrolló un taller en la comunidad de Guararí de Heredia, Costa Rica, titulado: Cada Pollo con su Rollo, donde participaron doce mujeres con edades entre los 12 y los 18 años y seis varones con edades entre los 10 y los 17 años. Empleando un lenguaje sencillo, se utilizó el juego cooperativo como metodología participativa, con el propósito de accesar a la información y a procesos de alfabetización informacional en materia de Derechos Humanos. Como resultado, los participantes identificaron la existencia de un conjunto de derechos plasmados en la Declaración Universal de los Derechos Humanos, que los faculta para vivir y para participar activamente en sus comunidades sin importar su condición económica, social y cultural.

Palabras Clave: Alfabetización Informacional; acceso a la Información; necesidades de información; usuarios; Declaración de Lyon sobre el Acceso a la Información y el Desarrollo; Guararí, Heredia; Costa Rica.

\section{ABSTRACT}

From the project of academical extension "Knowledge, Peace and Social Management" during the month of November of 2016 a workshop in the community of Guararía in Heredia, Costa Rica titled in Spanish as "Cada Pollo Con Su Rollo" was developed. In which twelve women in between the ages of 12 and 18 and six boys in between the ages 10 and 17 participated. Using simple language, the cooperative game was used as a participative methodology, with the purpose of accessing information and informal alphabetization processes in the subject of human rights. As a result, the participants identified the existence of a grouping of rights captured in the Universal Declaration of Human Rights, that empowers them to live and actively participate in their communities no matter what their economic, social and cultural condition might be.

Keywords: Information literacy; Access to information; Information needs; Users; Lyon Declaration on Access to Information and Development; Guararí, Heredia; Costa Rica.

1 Universidad Nacional de Costa Rica. Escuela de Bibliotecología Documentación e Información. COSTA RICA. https://orcid.org/0000-0002-4773-1359.1oirethc@gmail.com 


\section{INTRODUCCIÓN}

La relatoría especial para la libertad de expresión dice que: "el acceso a la información permite conocer qué derechos se tienen y cómo defenderlos" (Comisión Interamericana de Derechos Humanos, 2010, p. 9), en consencuencia, las bibliotecas se convierten en una importante puerta de acceso a la información porque ofrecen recursos y servicios adaptados a las necesidades de información de las comunidades a las que sirven.

Por lo anterior, la Escuela de Bibliotecología Documentación e Información de la Universidad Nacional de Costa Rica (UNA) participó durante tres años en el proyecto de extensión universitaria titulado: “Conocimiento, Paz y Gestión Social" ${ }^{2 \prime}$, centrada en el eje conocimiento y acceso a la información, cuyo propósito era contribuir con el logro de aprendizajes significativos, desde las experiencias y los saberes previos de las comunidades, sumando procesos de acceso y de alfabetización de la información que favorecieran el desarrollo y el fortalecimiento del conocimiento de las comunidades.

Durante este proyecto se detectaron necesidades de información en el campo de los Derechos Humanos que debían solventarse por medio del acceso a la información, pero de forma creativa y aceptada por un grupo de niños, niñas y jóvenes con edades entre los 10 y 18 años de edad ubicados en la comunidad de Guararí, Heredia, Costa Rica.

Para ello, se propuso un taller sobre la Declaración Universal de los Derechos Humanos, donde los participantes adquirieran conocimientos básicos de los artículos que conforman este instrumento internacional, a través de un proceso de alfabetización informacional usando el juego cooperativo: Cada Pollo con su Rollo, como método de acceso y construcción del conocimiento.

Por lo tanto, en el presente informe técnico se explican la experiencia, la metodología y un referente teórico que comprende los temas que están estrechamente relacionados con los procesos de acceso a la información, como por ejemplo, alfabetización informacional y necesidades de información de usuarios inmersos en sectores menos privilegiados de la sociedad costarricense, así como los postulados de la Declaración de Lyon sobre el acceso a la información y el desarrollo donde se identifica la intermediación de los bibliotecólogos con la información y las comunidades.

\section{USUARIO Y NECESIDADES DE INFORMACIÓN}

Según Monfasani y Curzel (2006), la literatura científica ha definido dos grupos de usuarios: los potenciales que requieren de información, pero no son conscientes de su necesidad, y los reales, quienes usualmente hacen uso de la información, ambos ejemplos existen en diversos contextos sociales, económicos y geográficos.

Asimismo, en la literatura científica aparecen diversos tipos de bibliotecas,

2 Este proyecto de extensión lo conforman las Escuelas de Bibliotecología, Documentación e Información, Escuela de Filosofía, y Escuela de Ecuménicas y Ciencias de la Religión de la Facultad de Filosofía y Letras de la Universidad Nacional (UNA). 
como por ejemplo, "biblioteca académica, escolar, especializada y pública" (Mosfasani y Curzel, 2006, p. 51), que atienden una diversidad de usuarios y sus necesidades de información. Las características de estos usuarios son:

1. Usuarios especializados en diversas áreas del conocimiento, como investigadores, académicos y empresarios, que requieren información científica para nutrir su quehacer.

2. Estudiantes de primaria, secundaria, universitarios de grado y postgrado, que necesitan información para elaborar trabajos de investigación en distintos niveles.

3. Usuarios que están fuera de los sistemas formales de educación, tal es el caso de amas de casa, trabajadores agrícolas y personas analfabetas.

Al respecto, Line (citado por González Teruel, 2005) "definió la necesidad de información como aquello que un individuo debería poseer para su trabajo, su investigación o su realización personal" (p. 71). No obstante, existe una diferencia entre la diversidad de usuarios y las necesidades de información que están estrechamente relacionadas con el trabajo que realizan, el ambiente donde se desenvuelven y el nivel de escolaridad que presenta cada usuario de la información; por eso, y a lo largo de su historia, las bibliotecas han utilizado el concepto de formación de usuarios para dar a conocer los servicios y los recursos disponibles, con el fin de satisfacer una necesidad de información detectada por un usuario que hace uso de la biblioteca.

Sin embargo, los individuos necesitan conocer las fuentes de información, desarrollar estrategias de búsqueda, comprender, usar y comunicar la información transformándola en conocimiento para su beneficio y el de muchas personas; a esto se le conoce como "alfabetización informacional" que iría más allá del concepto de formación de usuarios utilizado en las bibliotecas tradicionales.

\section{ACCESO Y ALFABETIZACIÓN DE LA INFORMACIÓN}

En la actualidad, las necesidades de información y la diversidad de fuentes de información son variadas a nivel de soporte y acceso. La búsqueda de información es relativamente fácil con el auge del desarrollo tecnológico, pero encontrar, evaluar y recuperar información para su utilización son tareas que requieren un proceso de alfabetización informacional previa, en ese sentido, Gómez Hernández y Benito Morales (2001) opinan que "el objetivo de la alfabetización en información es crear aprendices a lo largo de la vida, personas capaces para encontrar, evaluar y usar información eficazmente para resolver problemas o tomar decisiones, usando o sin usar una biblioteca" ( $p$. 56). Asimismo, existe el concepto Alfabetización Mediática e Informacional (AMI) que consiste en "empoderar a las personas para buscar, evaluar, utilizar y crear la información de una forma eficaz para alcanzar sus metas personales, sociales, ocupacionales y educativas" (UNESCO, 2011, p. 16).

Sin querer proponer una diferencia significativa entre ambos conceptos, interesa más para este abordaje teórico establecer que dicho concepto va más allá del uso y de la evaluación de fuentes de información, y cómo los individuos se apropian de la información, la transforman en conocimiento y ejercen procesos de comunicación. 
Ahora bien, para poder construir conocimiento en el proceso de alfabetización informacional se debe tener tanto acceso a la información como las competencias para analizarla, con el propósito de que los individuos puedan tomar decisiones informadas en el entorno donde se desarrollan.

Sin embargo, es importante tomar en cuenta que la sociedad está integrada por clases sociales o extremadamente ricas o tan pobres que no alcanzan a suplir sus necesidades básicas. En el caso de Costa Rica, esto se enfatiza en el último informe del Estado de la Nación, cuando señala que la "pobreza es la más grave expresión de la desigualdad, pues las personas que la sufren carecen de recursos suficientes para satisfacer un conjunto de necesidades que son básicas para vivir dignamente" (Programa Estado de la Nación, 2015, p. 49). Algo semejante sucede con el acceso a la información, porque es un bien que no está disponible para todos los sectores de la sociedad.

Por su parte, Gómez Hernández (2016) señala que: "el acceso a la información es un recurso transformacional y empoderador, especialmente de los que viven en situación de pobreza y marginación" (p. 84), por este motivo, la persona profesional en Bibliotecología se convierte en agente social de cambio, porque contribuya al acceso de la información y a procesos de alfabetización informacional, que favorecen la construcción del conocimiento desde las propias condiciones de los usuarios y sus comunidades.

Por lo tanto, para que exista un desarrollo equitativo en la sociedad se deben reducir la brecha económica, social y cultural entre los grupos minoritarios, esto es migrantes, personas refugiadas, personas con discapacidades, personas adultas mayores, población infantil y jóvenes, así como lograr un adecuado acceso a la información, convirtiendo a la biblioteca en un espacio social de intercambio de conocimiento, mediante juegos cooperativos y experiencias aportadas por cada integrante de la comunidad con lenguaje sencillo, accesible y de fácil uso para que no exista distinción del nivel educativo, social o cultural.

\section{DECLARACIÓN DE LYON SOBRE EL ACCESO A LA INFORMACIÓN Y EL DESARROLLO}

La Declaración de Lyon (Federación Internacional de Asociaciones de Bibliotecarios y Bibliotecas [IFLA], 2014) indica que para que exista un desarrollo sostenible y para que los individuos tengan una mejor calidad de vida debe existir un progresivo acceso a la información y al conocimiento con la ayuda de las tecnologías.

Por eso, dentro de los postulados de la Declaración figura uno que toca el quehacer de las personas profesionales en Bibliotecología de forma medular:

Los intermediarios de la información, como las bibliotecas, archivos, organizaciones de la sociedad civil, líderes comunitarios y medios de comunicación tienen la capacidad y recursos para ayudar a los gobiernos, instituciones e individuos a transmitir, organizar, estructurar y comprender la información que es importante para el desarrollo. (IFLA, 2014, p. 2)

Dicha intermediación invita a la persona competente a tener una proyección más significativa en la sociedad, es decir, ya no basta el diseño de estrategias 
de búsqueda y la disponibilidad de información científica, muchas veces en lenguaje poco comprensible para las comunidades vulnerables que apenas consiguen leer y escribir; por eso, cada profesional en el área de la información debe seleccionar los recursos y los medios de acceso a la información según las condiciones propias de cada usuario.

Por consiguiente, los procesos de acceso y de alfabetización informacional deben ser planificados de acuerdo a las condiciones económicas, sociales y culturales de las comunidades, tomando en consideración sus niveles de escolaridad, necesidades de información, proponiendo diversos soportes y formas de acceso, convirtiendo, por ejemplo, a las imágenes y a los juegos cooperativos en medios que permitan el acceso y propiciar el proceso de alfabetización informacional en las comunidades menos favorecidas.

\section{LOS JUEGOS COOPERATIVOS Y EL ACCESO A LA INFORMACIÓN}

El juego cooperativo "promueve un entorno de aprendizaje vivencial, participativo, reflexivo y crítico hacia la transformación personal o grupal para incidir en lo social" (Cerdas Agüero, 2013, p. 109), favoreciendo el análisis, la aceptación y el respeto de las opiniones de sus iguales, pues conlleva la construcción de acuerdos y la resolución de situaciones particulares planteadas desde los diversos entornos donde se desarrollan los participantes.

\section{Al respecto, Arranz Beltrán (1993) señala que}

los juegos cooperativos tratan de no excluir ni humillar a nadie, de conseguir diversión sin tener la amenaza de no conseguir el objetivo marcado, y de favorecer un ambiente de aprecio recíproco donde no se mira a la otra como competidora sino como compañera de juego. (p. 8)

Por tal motivo, los mecanismos de los juegos cooperativos son la colaboración, la construcción de conocimiento, la participación y el aprendizaje de cada integrante del grupo, sin que medie la descalificación por sus opiniones, habilidades y destrezas.

Entonces, el juego cooperativo se convierte en un vehículo para acceder a la información suministrada durante la actividad, beneficiando el acceso y la alfabetización informacional, favoreciendo la habilidad de identificar necesidades de información, fuentes donde satisfagan sus necesidades, cómo evaluarlas, analizarlas, convertirlas en conocimientos y comunicarlas desde un enfoque lúdico, participativo y respetuoso.

\section{METODOLOGÍA UTILIZADA EN EL DESARROLLO DEL}

\section{TALLER}

La metodología fue participativa en el proceso de alfabetización informacional por medio de un taller dedicado al acceso a la información en Derechos Humanos impartida en Guararí, en la provincia de Heredia, Costa Rica, en 2016. 
El propósito del taller fue dar herramientas de acceso a fuentes y conseguir un conocimiento básico de la Declaración Universal de los Derechos Humanos utilizando lenguaje sencillo y con la ayuda de los juegos cooperativos. Para esto, se usó la investigación-acción que

considera la situación desde el punto de vista de los participantes, describirá y explicará lo que sucede con el mismo lenguaje utilizado por ellos; o sea con el lenguaje de sentido común que la gente usa para describir las situaciones sociales de la vida diaria. (Elliot, 1990, p. 27)

De hecho, la investigación-acción permitió describir una serie de actividades realizadas por los facilitadores del taller, y cada participante tuvo la oportunidad de sentirse en libertad de formar parte del proceso, ya que estas actividades tienen en común la identificación de acciones ejecutadas por los participantes, la observación y reflexión del proceso de aprendizaje.

En ese sentido, para Latorre (2004), la investigación-acción está constituida por los siguientes ciclos o fases:" plan de acción, acción, observar y reflexionar" (p. 21). A continuación, se explicarán las primeras tres fases desarrolladas en el taller sobre los Derechos Humanos en la comunidad de Guararí, Heredia, y la reflexión se explica en los resultados del informe técnico.

\section{Fase 1. Plan de acción}

El plan de acción permitió a quienes facilitaron el taller seleccionar la comunidad, la población y el recurso de información que permitiría dar acceso a la Declaración Universal de los Derechos Humanos, esta fue una comunidad de la zona de Guararí considerada urbano marginal, con población migrante nicaragüense, en condiciones de pobreza, riesgo social y localizada en el distrito de San Francisco, del cantón Central de la provincia de Heredia.

La población que asistió estuvo conformada por doce mujeres con edades entre los 12 y 18 años, y seis varones con edades entre los 10 y los 17 años, estudiantes del sistema formal de educación. Las niñas y los niños asisten a la Escuela de la Finca, ubicada en la zona de Guararí, y los adolescentes acuden al Colegio de los Lagos de Heredia; además, integran un grupo organizado del Centro Cívico para la Paz de Guararí con el cual el proyecto de extensión universitaria “Conocimiento, Paz y Gestión Social” trabajó durante el 2016.

Para el desarrollo del taller se seleccionó como fuente de información la Declaración Universal de los Derechos Humanos, adaptada por Limpens y Nava (2011), con la actividad: Cada Pollo con su Rollo, que pertenece a una serie de juegos cooperativos adaptados a un lenguaje sencillo que puede ser interiorizado por individuos de diversas edades sin conocimientos previos en el derecho internacional sobre los Derechos Humanos.

El juego está conformado por 60 tarjetas de las cuales 30 contienen los artículos de la Declaración Universal de los Derechos Humanos, y las otras 30 los dibujos que representan este instrumento internacional. El juego cooperativo Cada Pollo con su Rollo y las instrucciones para el desarrollo del juego están disponibles en Limpens y Nava (2011).

\section{Fase 2. Acción}

El propósito fue generar una serie de interrogantes, crear una necesidad de información y dar acceso a una fuente de información por medio del juego 
cooperativo Cada Pollo con su Rollo. Para desarrollarlo, se realizaron las siguientes actividades:

- En primer lugar, se hicieron preguntas generadoras: ¿Qué son los Derechos Humanos? ¿Han escuchado hablar de la Declaración Universal de los Derechos Humanos? ¿Qué es la Declaración Universal de los Derechos Humanos? ¿Dónde se puede encontrar información sobre el tema? Estas interrogantes permitirían identificar una necesidad de información entre los participantes.

- En segundo, los participantes se dividieron en dos grupos, por edad, se les entregó un recurso de información, es decir, una tarjeta con un dibujo, para que analizaran e identificaran qué derecho representaba esa imagen.

- Tercero, una vez concluida la fase de identificación y de análisis debían encontrar el texto que representaba el dibujo de la Declaración Universal de los Derechos Humanos.

- Cuarto, cada participante presentó ante el grupo el texto y mostró el dibujo que lo representaba.

- En quinto lugar, realizaron un dibujo que simbolizara alguno de los derechos representados en este instrumento internacional, a partir de su percepción de vida.

- Por último, los grupos reflexionaron para establecer una serie de conclusiones en relación con el proceso de aprendizaje conseguido en el taller.

\section{Fase 3. Observar}

En esta fase, los personas facilitadoras observaron el proceso de análisis y reflexión generado en los grupos de trabajo, lo que permitió recopilar sus expresiones y presentar los resultados obtenidos durante el taller.

\section{FASE DE REFLEXIÓN Y RESULTADOS}

De las preguntas generadoras se obtuvieron las siguientes respuestas:

- Algunos habían escuchado hablar de la Declaración Universal de los Derechos Humanos y lo relacionaban como un grupo de derechos que tienen las personas que viven en un determinado país.

- Ninguno de los participantes había tenido acceso a la Declaración, desconocían el contenido de sus artículos y cómo debían formar parte de sus vidas.

- Como fuentes de información para tener acceso a la información señalaron la Internet y las bibliotecas.

En el proceso de análisis y reflexión señalaron qué significado podrían tener algunas de las imágenes para cada participante, por eso, en la Tabla 1 se describen algunas de esas interpretaciones generadas en un lenguaje sencillo. 
TABLA 1

Interpretación de los participantes del taller Cada Pollo con su Rollo, de acuerdo a los artículos de la Declaración Universal de los Derechos Humanos. Guararí, Heredia, Costa Rica, 2016

\section{ARTÍCULOS DE LA DECLARACIÓN \\ UNIVERSAL DE LOS DERECHOS HUMANOS INTERPRETACIÓN DE LOS PARTICIPANTES (LIMPENS Y NAVA, 2011)}

Artículo 3: Tú tienes derecho a la vida y a vivir en libertad en condiciones de seguridad.

Artículo 4: Nadie tiene derecho de hacer de tí su esclavo y tú no debes de hacer de nadie tu esclavo.

Artículo 5: Nadie tiene derecho a torturarte.

Artículo 9: Nadie tiene derecho a enviarte a la cárcel, a mantenerte en ella o a enviarte fuera de tu país injustamente o sin razón válida.

Artículo 16: Tan pronto como una persona llega a la edad prevista por la ley, tiene derecho a casarse y fundar una familia. Al casarte, no tiene ninguna importancia el color de tu piel, el país de que procedes ni tu religión. Los hombres y las mujeres tienen los mismos derechos cuando están casados y también cuando están separados. Nadie debe obligar a una persona a casarse. El gobierno de tu país debe proteger a toda tu familia.

Artículo 17: Tienes derecho a poseer tus propios bienes y nadie tiene derecho a despojarte de ellos sin una razón válida.

Artículo 23: Tienes derecho a trabajar, a elegir libremente tu trabajo, y a percibir un salario que te permita vivir y mantener a tu familia. Si un hombre y una mujer realizan el mismo trabajo, deben percibir la misma paga. Todas las personas tienen derecho a asociarse para defender sus intereses.

Artículo 26: Tienes derecho a asistir a la escuela y todos deben ir a ella. La enseñanza primaria debe ser gratuita. Tienes derecho a aprender un oficio o a continuar tus estudios mientras lo desees. En la escuela debes poder desarrollar tus aptitudes y te deben enseñar a llevarte bien con los demás, cualquiera sea tu raza, religión o el país de que procedas. Tus padres tienen el derecho de elegir cómo y qué se te ha de enseñar en la escuela
"Tenemos derecho a caminar libremente por nuestro barrio".

"Nadie puede tenernos atrapados en ningún lugar".

"Nadie tiene derecho a maltratarnos ni abusar de nosotros".

"Nadie tiene derecho a encerrarnos ¡Si no hemos hecho nada malo!".

"Tienes derecho a tener una pareja y familia si así lo queremos".

"Tienes derecho a tener una casa".

"Tienes derecho a tener un trabajo que nos permita vivir con comodidad".

"Todos tenemos derecho a estudiar".

Fuente: Elaboración de la autora.

La interpretación hecha de cada una de las ilustraciones no se aleja de la esencia de los artículos que conforman la Declaración Universal de los Derechos Humanos, que representa apropiación significativa de conocimientos a través de los instrumentos empleados por los participantes del taller. 
El proceso de alfabetización informacional otorga a los individuos la facultad de buscar, evaluar, utilizar y crear información para sus necesidades de información. La preparación del taller Cada Pollo con su Rollo permitió dar acceso a la información por medio del juego cooperativo y crear un ambiente de aprendizaje usando un lenguaje sencillo que provocó en los participantes una apropiación de la información y un proceso de reflexión de cada uno de los artículos de la Declaración, tal y como lo señala Mujica (2002) "el sujeto es el principal constructor del conocimiento y que construye significados cuando hace uso de experiencias y conocimientos previos, cuando tiene interés y disponibilidad y cuando recibe la orientación oportuna y efectiva" (p. 6).

Al finalizar el taller, los participantes identificaron la existencia de un conjunto de derechos plasmados en la Declaración Universal de los Derechos Humanos, que los faculta para vivir y participar activamente en sus comunidades sin importar su condición económica, social y cultural.

De esta forma, el proceso de alfabetización informacional facultó a cada participante a accesar, analizar, comprender y comunicar información, contribuyendo de manera significativa al desarrollo de una población joven empoderada y crítica de sus derechos y obligaciones en sus comunidades y en la sociedad.

\section{CONCLUSIÓN}

El proceso de alfabetización informacional debe ir más allá de las cuatro paredes de las bibliotecas, debe llegar a las comunidades en riesgo social, con fuentes y formas de acceso que se adapten a sus condiciones económicas, sociales y culturales. Asimismo, el acceso a la información y el intercambio de conocimientos previos de las comunidades contribuyen de manera significativa al fortalecimiento de su desarrollo.

Por consiguiente, el taller desarrollado en Guararí favoreció el proceso de alfabetización informacional de los participantes, motivando y transmitiendo la pasión por aprender, generando receptores críticos capaces de identificar sus derechos y sus obligaciones en sus comunidades. Además, a través del juego cooperativo los participantes tuvieron acceso a la información, compartieron experiencias, colaboraron entre sí y establecieron un vínculo de cooperación y de solidaridad.

Por su carácter lúdico y motivador, el juego sirvió para estimular a los participantes, fortaleciendo el proceso de acceso y alfabetización informacional que tenía como objetivo el taller Cada Pollo con su Rollo, al mismo tiempo, fue posible abordar algunos de los artículos de la Declaración Universal de los Derechos Humanos, dando lugar a un proceso de alfabetización informacional donde la población reconoció una necesidad de información en torno a ¿cuáles son sus derechos?, y ¿dónde pueden identificarlos? De esta manera, los procesos de alfabetización informacional deben buscar, como fin último, el uso efectivo de la información para la generación de conocimiento y la comunicación. 


\section{REFERENCIAS}

ArranzBeltrán,E.(1993).Juegoscooperativosysincompeticiónparalaeducación infantil. Recuperado de http://www.educacionfisicaenprimaria.es/ uploads/4/2/1/3/4213158/_juegos cooperativos y sin competicion para infantil.pdf

Cerdas Agüero, E. (2013). Experiencias y aprendizajes con juegos cooperativos. Revista de Paz y Conflictos, 6, 107-123

Comisión Interamericana de Derechos Humanos. (2010). El derecho de acceso a la información en el marco jurídico interamericano. Recuperado de http://www.oas.org/es/cidh/expresion/docs/publicaciones/ACCESO\%20 A\%20LA\%20INFORMACION\%20FINAL\%20CON\%20PORTADA.pdf

Ministerio de Justicia y Paz (2017). Centros Cívicos por la Paz. Recuperado de http://www.mjp.go.cr/viceministeriopaz/CentroCivico

Elliot, J. (1990). La investigación-acción en educación. Madrid: Morata

Latorre, A. (2004). La investigación Acción y conocer y cambiar la práctica educativa. Barcelona. Editorial Graó.

Limpens, F., y Nava, M. (2011). Cada Pollo Con Su Rollo: la Declaración Universal de los Derechos Humanos para niñ@s. Recuperado de https://www.iidh. ed.cr/multic/UserFiles/Biblioteca/IIDH/10_2011/1732.pdf

Gómez Hernández, J. (2016). Usuarios en vulnerabilidad social: ¿por qué y para qué generar capital cultural desde la biblioteca? Anuario ThinkEPI, $10,83-93$.

Gómez Hernández, J., y Benito Morales, F. (2001). De la formación de usuarios a la alfabetización informacional: propuestas para enseñar las habilidades de información. Scire, 7(2), 53-83

GonzálezTeruel, A. (2005). Los estudios de necesidadesy usos de la información: fundamentos y perspectivas actuales. España: Ediciones Trea.

Federación Internacional de Asociaciones de Bibliotecarios y Bibliotecas. (2014). Declaración de Lyon sobre el acceso a la información y el desarrollo. Recuperado de http://www.lyondeclaration.org/content/ pages/lyon-declaration-es-v2.pdf

Monfasani, R., y Curzel, M. (2006). Usuarios de la información: formación y desafíos. Buenos Aires: Alfagrama.

Mujica, R. (2002). La metodología de la educación en derechos humanos. San José, Costa Rica: IIDH

Programa Estado de la Nación. (2015). Vigésimo primer Informe Estado de la Nación en Desarrollo Humano Sostenible. Recuperado de http://www. estadonacion.or.cr/21/assets/pen-21-2015-baja.pdf

UNESCO. (2011). Alfabetización mediática e informacional: curriculum para profesores. Paris: UNESCO. 


\section{e $\begin{aligned} & \text { e-Ciencias de la } \\ & \text { Información }\end{aligned}$}
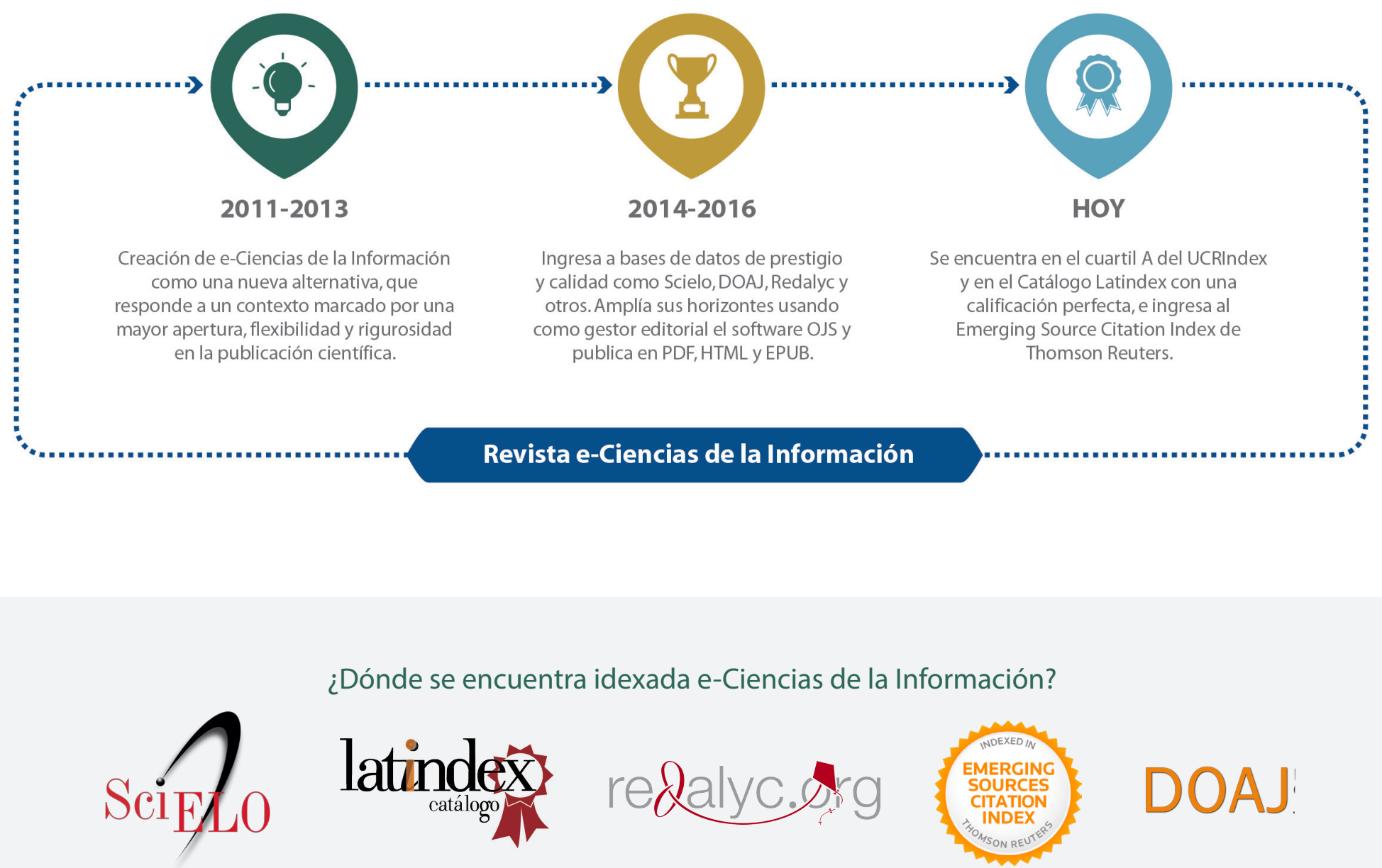

¿Dónde se encuentra idexada e-Ciencias de la Información?
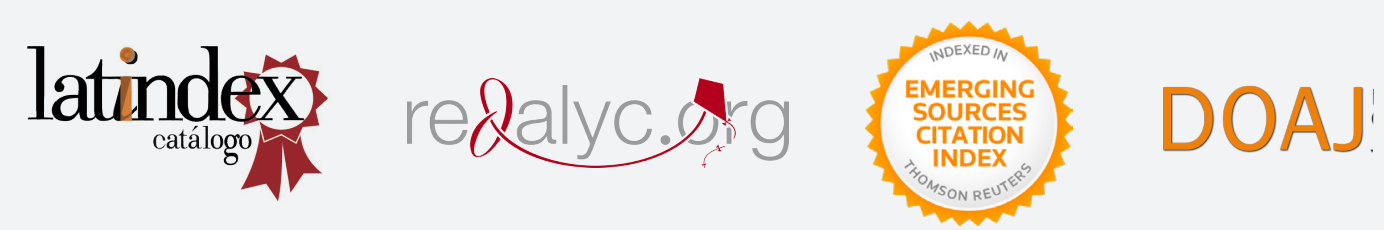

Para más información ingrese a nuestra lista completa de indexadores

¿Desea publicar su trabajo?

Ingrese aquí

O escríbanos a la siguiente dirección

revista.ebci@ucr.ac.cr 